This item was submitted to Loughborough's Research Repository by the author.

Items in Figshare are protected by copyright, with all rights reserved, unless otherwise indicated.

\title{
Vortex in the charged Bose liquid
}

PLEASE CITE THE PUBLISHED VERSION

PUBLISHER

(C) American Physical Society

LICENCE

CC BY-NC-ND 4.0

REPOSITORY RECORD

Alexandrov, A.S.. 2019. "Vortex in the Charged Bose Liquid". figshare. https://hdl.handle.net/2134/1318. 


\title{
Vortex in the charged Bose liquid
}

\author{
A. S. Alexandrov \\ Department of Physics, Loughborough University, Loughborough LE11 3TU, United Kingdom
}

(Received 29 June 1999)

\begin{abstract}
Equations describing a single vortex in the charged Bose liquid at zero temperature are derived. The zero-temperature coherence length, magnetic-field penetration depth, vortex structure and energy, and lower critical field are calculated. The vortex differs from that in type-II BCS superconductors or in neutral superfluids. Its core is charged, and there is an electric field inside the core. [S0163-1829(99)00845-0]
\end{abstract}

A long time ago Schafroth $^{1}$ demonstrated that a noninteracting gas of charged bosons exhibits the MeissnerOchsenfeld effect below the ideal Bose-gas condensation temperature. Later on, the one-particle excitation spectrum of the Coulomb Bose gas of high density was calculated by Foldy, ${ }^{2}$ who worked at zero temperature using the Bogoliubov $^{3}$ approach. The Bogoliubov method leads to the result that the elementary excitations of the system have, for small momenta, energies characteristic of plasma oscillations. The subsequent studies were concerned with the critical exponents and the change in the transition temperature from that of the ideal gas, ${ }^{4,5}$ with the random-phase approximation (RPA) dielectric response function and screening, ${ }^{6}$ and with the theory of the charged Bose liquid beyond the lowest-order Bogoliubov approximation. ${ }^{7-9}$ We found the upper critical magnetic field $H_{c 2}(T)$, below which charged bosons are condensed, ${ }^{10}$ and derived the BogoliubovdeGenes $(\mathrm{BdG})$-type equations describing charged bosons in the inhomogeneous electromagnetic field. ${ }^{11,12}$ The predicted temperature dependence of $H_{c 2}(T)$ was observed in many high- $T_{c}$ cuprates. $^{13}$ The magnetic-field dependence of the specific heat ${ }^{14}$ also appeared to be consistent with that expected for the charged Bose gas. ${ }^{15}$ Recently, a parameter-free expression for the condensation temperature of charged bosons on a lattice has been derived, which describes the critical temperature of a few dozen cuprates without any fitting parameters. ${ }^{16,17}$ These and many other experimental observations point uniquely to the possibility of an interesting electronic state in solids, which is a charged Bose liquid (CBL) of preformed pairs, presumably bipolarons. ${ }^{18,19} \mathrm{Be}-$ sides this "application'" aspect, charged bosons represent a fundamental reference system of the condensed-matter physics. As follows from our analysis of the upper critical field ${ }^{10}$ and of the specific heat ${ }^{15} \mathrm{CBL}$ does not share the critical behavior of the BCS superfluids nor the universal [threedimensional (3D)] $x-y$ properties of neutral superfluids like ${ }^{4} \mathrm{He}$ but exhibits an unusual critical behavior.

In this paper I formulate and solve the equations describing a single vortex in $\mathrm{CBL}$ at $T=0 \mathrm{~K}$. The vortex has a structure different from that in the BCS and neutral superfluids. Its core is charged and there is an electric field inside the core.

The Hamiltonian of charged bosons on a chargecompensating homogeneous background (to ensure global charge neutrality) in an external magnetic field with the vector potential $\mathbf{A}(\mathbf{r})$ is given by

$$
\begin{aligned}
H= & \int d \mathbf{r} \psi^{\dagger}(\mathbf{r})\left[-\frac{\left(\hbar \nabla-i e^{*} \mathbf{A} / c\right)^{2}}{2 m^{*}}-\mu\right] \psi(\mathbf{r}) \\
& +\frac{1}{2} \int d \mathbf{r} \int d \mathbf{r}^{\prime} V\left(\mathbf{r}-\mathbf{r}^{\prime}\right)\left[\psi^{\dagger}(\mathbf{r}) \psi^{\dagger}\left(\mathbf{r}^{\prime}\right) \psi\left(\mathbf{r}^{\prime}\right) \psi(\mathbf{r})\right. \\
& \left.-2 n \psi^{\dagger}(\mathbf{r}) \psi(\mathbf{r})\right],
\end{aligned}
$$

where $m^{*}, e^{*}, n, \mu$ are the mass, charge, average density, and the chemical potential of bosons, and $V(\mathbf{r})=e^{* 2} / \epsilon_{0}|\mathbf{r}|$ is the Coulomb repulsion with $\epsilon_{0}$ the static dielectic constant of a host material.

The equation of motion for the field operator $\psi$ is derived using this Hamiltonian. If the density is relatively high, so the dimensionless Coulomb repulsion $r_{s}$ $=m^{*} e^{* 2} / \hbar^{2} \epsilon_{0}(4 \pi n / 3)^{1 / 3}$ is not very large, one can expect that the occupation numbers of one-particle states are not very much different from those in the ideal Bose gas. In particular, one state remains to be macroscopically occupied at $T=0 \mathrm{~K}$. Then, following Bogoliubov ${ }^{3}$ one can separate the large matrix element $\psi_{0}$ from $\psi$ by treating the rest $\widetilde{\psi}$ as small fluctuations:

$$
\psi(\mathbf{r}, t)=\psi_{0}(\mathbf{r}, t)+\widetilde{\psi}(\mathbf{r}, t) .
$$

The anomalous average $\psi_{0}(\mathbf{r}, t)=\langle\psi(\mathbf{r}, t)\rangle$ is equal to $\left(n_{0}\right)^{1 / 2}$ in a homogeneous system, where $n_{0}$ is the condensate density.

Substituting the Bogoliubov displacement transformation Eq. (2) into the equation of motion and collecting $c$-number terms of $\psi_{0}$, we obtain a set of the BdG-type equations. ${ }^{12}$ The macroscopic condensate wave function (the order parameter) obeys the following equation:

$$
\begin{aligned}
i \hbar \frac{\partial}{\partial t} \psi_{0}(\mathbf{r}, t)= & \left.-\frac{\left(\hbar \nabla-i e^{*} \mathbf{A} / c\right)^{2}}{2 m^{*}}-\mu\right] \psi_{0}(\mathbf{r}, t) \\
& +\int d \mathbf{r}^{\prime} V\left(\mathbf{r}-\mathbf{r}^{\prime}\right) \\
& \times\left[\psi_{0}^{*}\left(\mathbf{r}^{\prime}, t\right) \psi_{0}\left(\mathbf{r}^{\prime}, t\right)-n\right] \psi_{0}(\mathbf{r}, t) .
\end{aligned}
$$

There are also higher-order terms in $r_{s}$ proportional to the density of bosons, $\widetilde{n} \simeq 0.2 n r_{s}^{3 / 4}$, pushed up from the condensate by the Coulomb interaction at $T=0 \mathrm{~K} .^{2}$ They are negligible even for the intermediate value of $r_{s} \simeq 1$. With the same accuracy one can take $n_{0}=n$. 
The integro-differential Eq. (3) is essentially different from the Ginsburg-Landau ${ }^{20}$ and the Gross-Pitaevskii ${ }^{21,22}$ equations, describing the order parameter in the BCS and neutral superfluids, respectively. As recognized by London ${ }^{23}$ and discussed in detail by Vinen ${ }^{24}$ superconducting metals and neutral superfluids $\left({ }^{4} \mathrm{He}\right)$ have many features in common with their characteristic properties resulting from the macroscopic quantum coherence and a short-range (local) interaction. While CBL shares the quantum coherence owing to the Bose-Einstein condensate (BEC), the long-range (nonlocal) interaction leads to a few peculiarities. It is worthwhile to emphasize that one should apply a bare (unscreened) Coulomb repulsion in Eq. (3) to avoid the double counting of the interaction in the perturbation theory diagrams. ${ }^{11}$ Actually, the coherence length appears to be just the same as the screening radius (see below).

Under the stationary conditions, Eq. (3) supplemented by the current density, $\mathbf{j}(\mathbf{r})=\left(e^{*} / m^{*}\right) \operatorname{Re}\left[\psi_{0}^{*}(\mathbf{r})(-i \hbar \nabla\right.$ $\left.\left.-e^{*} \mathbf{A} / c\right) \psi_{0}(\mathbf{r})\right]$, and by the Maxwell equations, is reduced to a set of three nonlinear differential equations:

$$
\begin{gathered}
\frac{1}{\kappa^{2} \rho} \frac{d}{d \rho} \rho \frac{d f}{d \rho}-\frac{1}{f^{3}}\left(\frac{d h}{d \rho}\right)^{2}-\phi f=0, \\
\frac{1}{\kappa^{2} \rho} \frac{d}{d \rho} \rho \frac{d \phi}{d \rho}=1-f^{2},
\end{gathered}
$$

and

$$
\frac{1}{\rho} \frac{d}{d \rho} \frac{\rho}{f^{2}} \frac{d h}{d \rho}=h
$$

As usual ${ }^{25}$ these equations are written in a form which introduces the dimensionless quantities: $\left|\psi_{0}\right|=n_{0}^{1 / 2} f, \quad \mathbf{r}$ $=\lambda(0) \rho, e^{*} \xi(0) \lambda(0) \operatorname{curl} \mathbf{A} / \hbar c=\mathbf{h}$ for the order parameter, length, and magnetic field, respectively. The electric field potential given by

$$
\phi=\frac{1}{e^{*} \phi_{c}} \int d \mathbf{r}^{\prime} V\left(\mathbf{r}-\mathbf{r}^{\prime}\right)\left[\left|\psi_{0}\left(\mathbf{r}^{\prime}\right)\right|^{2}-n\right]
$$

with a fundamental unit $\phi_{c}=\hbar^{2} / 2 e^{*} m^{*} \xi(0)^{2}$. The coherence length is about the same as the RPA screening radius at $T=0 \mathrm{~K},{ }^{6} \xi(0)=\left(\hbar / 2^{1 / 2} m^{*} \omega_{p 0}\right)^{1 / 2}$, where $\omega_{p 0}$ $=\left(4 \pi n e^{* 2} / \epsilon_{0} m^{*}\right)^{1 / 2}$ is the zero-temperature plasma frequency. ${ }^{2}$ The London penetration depth is $\lambda(0)$ $=\left(m^{*} c^{2} / 4 \pi n e^{* 2}\right)^{1 / 2}$. Cylindrical symmetry is assumed.

There are six boundary conditions in a single-vortex problem. Four of them are the same as in the BCS superconductor: ${ }^{26} h=d h / d \rho=0, f=1$ for $\rho=\infty$ and the flux quantization condition, $d h / d \rho=-p f^{2} / \kappa \rho$ for $\rho=0$, where $p$ is an integer. The remaining two conditions are derived from the global charge neutrality: $\phi=0$ for $\rho=\infty$ and

$$
\phi(0)=\int_{0}^{\infty} \rho \ln (\rho)\left(1-f^{2}\right) d \rho
$$

for the electric field at the origin. Besides of that the chemical potential $\mu$ is zero, as it should be in the globally neutral
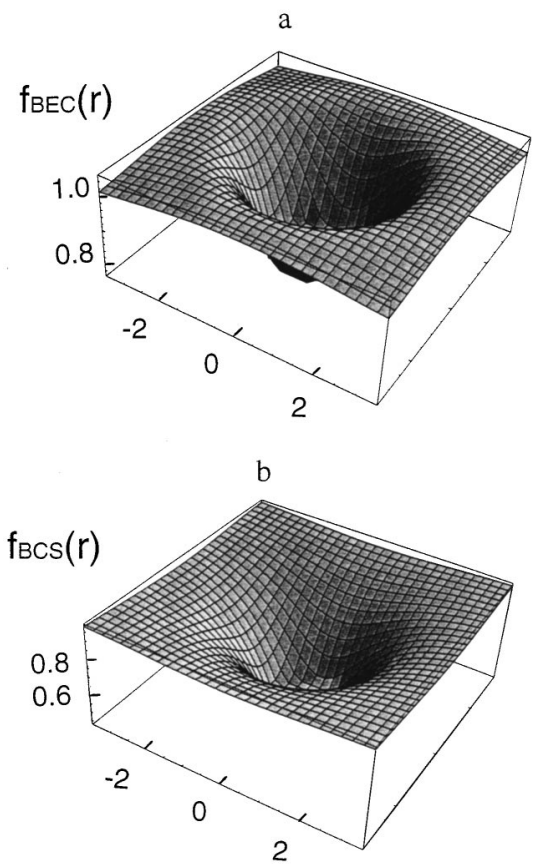

FIG. 1. Vortex core profile in the charged Bose liquid, $f_{\mathrm{BEC}}$, (a) compared with the vortex in the BCS superconductors, $f_{\mathrm{BCS}}$, (b).

CBL in the thermal equilibrium below the BEC critical temperature.

The most realistic regime of CBL is an extreme type II with a very large Ginsburg-Landau parameter, $\kappa$ $=\lambda(0) / \xi(0) \gg 1$ (see below). In this regime Eq. (6) is reduced to the London equation with the familiar solution, ${ }^{26}$ $h=p K_{0}(\rho) / \kappa$ where $K_{0}(\rho)$ is the Hankel function of imaginary argument of zero order. For the region $\rho \leqslant p$, where the order parameter and the electric field differ from unity and zero, respectively, one can use the flux quantization condition to integrate out the magnetic field. That leaves us with the two parameter-free equations written for $r=\kappa \rho$ as

$$
\frac{1}{r} \frac{d}{d r} r \frac{d f}{d r}-\frac{p^{2} f}{r^{2}}-\phi f=0
$$

and

$$
\frac{1}{r} \frac{d}{d r} r \frac{d \phi}{d r}=1-f^{2} .
$$

They are satisfied by regular solutions of the form $f=c_{p} r^{p}$ and $\phi=\phi(0)+\left(r^{2} / 4\right)$ when $r \rightarrow 0$. The constants $c_{p}$ and $\phi(0)$ are determined by complete numerical integration of Eqs. (9) and (10). The numerical results for $p=1$ are shown in Figs. 1 and 2 with $c_{1} \simeq 1.5188$ and $\phi(0) \simeq-1.0515$.

In the region $p \ll r<p \kappa$ the solutions are $f=1$ $+\left(4 p^{2} / r^{4}\right)$ and $\phi=-p^{2} / r^{2}$. In this region the solution for $f$ differs qualitatively from the BCS order parameter, ${ }^{25} f_{\mathrm{BCS}}$ $=1-\left(p^{2} / r^{2}\right)$ (see also Fig. 1), which is a result of the local charge redistribution caused by the magnetic field. Different from the BCS superconductor, where the total density of electrons remains constant across the sample, CBL allows for the flux penetration by redistributing the density of 


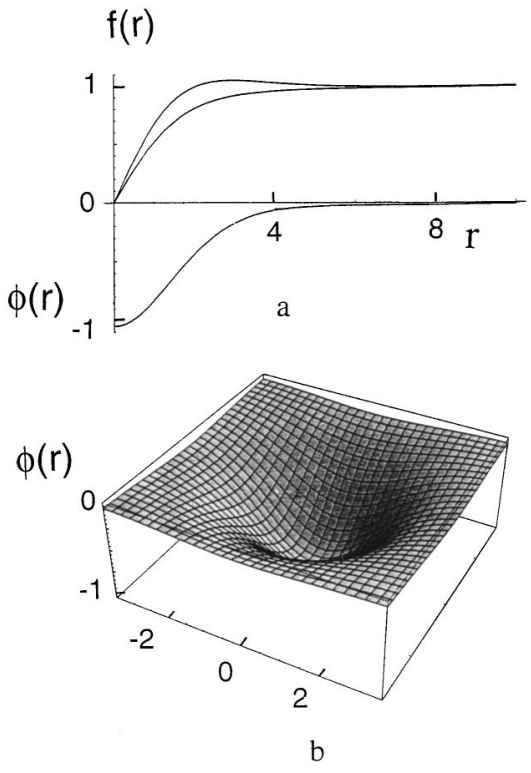

FIG. 2. Electric-field potential $\phi$ as a function of the distance [measured in units of $\xi(0)$ ] (lower curve) together with the CBL (upper curve) and BCS order parameters (a); its profile is shown in (b).

bosons within the coherence volume. This leads to an increase of the order parameter compared with the homogeneous case $(f>1)$ in the region close to the vortex core. Inside the core the order parameter is suppressed, Fig. 1, as in the BCS superconductors. The resulting electric field, Fig. 2(b), (together with the magnetic field) acts as an additional centrifugal force increasing the steepness $\left(c_{p}\right)$ of the order parameter compared with the BCS superfluid where $c_{1}$ $\simeq 1.1664$, Fig. 2(a).

The breakdown of the local charge neutrality is due to the absence of any equilibrium normal-state solution in CBL below $H_{c 2}(T)$ line. Both superconducting $(\Delta \neq 0)$ and normal $(\Delta=0)$ solutions are allowed at any temperature in the BCS superconductors. Then the system decides which of two phases (or their mixture) is energetically favorable, so that the local charge neutrality is respected providing the minimum of free energy. ${ }^{27}$ In contrast, an equilibrium normalstate solution of the complete set of the BdG equations (with $\psi_{0}=0$ ) does not exist in CBL because it does not respect the density sum rule below $H_{c 2}(T) .{ }^{10}$ Hence there are no different phases to mix, and the only way to aquire a flux in the thermal equilibrium is to redistribute the local density of bosons at the expence of their Coulomb energy. This energy determines the vortex free energy $\mathcal{F}=E_{\mathrm{V}}-E_{0}$, which is the difference of the energy of CBL with, $E_{\mathrm{v}}$, and without, $E_{0}$, the magnetic flux:

$$
\begin{aligned}
\mathcal{F}= & \int d \mathbf{r}\left(\frac{1}{2 m^{*}}\left|\left(\hbar \nabla-i e^{*} \mathbf{A} / c\right) \psi_{0}\right|^{2}+\frac{e^{*}}{2} \phi_{c} \phi\left(\left|\psi_{0}\right|^{2}-n\right)\right. \\
& \left.+\frac{(\operatorname{curl} \mathbf{A})^{2}}{8 \pi}\right) .
\end{aligned}
$$

By the use of Eqs. (4) and (6) it can be written in the dimensionless form as

$$
F=2 \pi \int_{0}^{\infty}\left(h^{2}-\frac{1}{2} \phi\left(1+f^{2}\right)\right) \rho d \rho .
$$

In the large $\kappa$ limit the main contribution comes from the region $p / \kappa<\rho<p$, where $f \simeq 1$ and $\phi \simeq-p^{2} / \kappa^{2} \rho^{2}$; the energy is thus the same as that in the BCS superconductor, $F$ $\simeq 2 \pi p^{2} \ln (\kappa) / \kappa^{2}$. It is seen that the most stable solution is the formation of the vortex with one flux quantum, and the lower critical field is the same as in the BCS superconductor, ${ }^{26}$ $h_{c 1} \simeq \ln (\kappa) / 2 \kappa$. However, differently from the BCS superconductors, where the Ginsburg-Landau phenomenology is microscopically derived by Gor' $\mathrm{kov}^{28}$ in the temperature region close to $T_{c}$, the CBL vortex structure is derived here in the low-temperature region. The zero temperature solution, Fig. 2, is actually applied in a wide temperature range well below the Bose-Einstein condensation temperature, where the depletion of the condensate remains small. Normal bosons pushed up from the condensate will be inhomogeneously distributed across the sample with the maximum density in the vortex core, where their potential energy in the electric field $\phi$ is at minimum. The excess density of normal bosons inside the core, $\tilde{n}$, is determined by the balance between the potential energy in the field $\phi$, which is about $-\tilde{n} \pi \xi^{2} L e^{*} \phi_{c}$ and their Coulomb repulsive energy about $\left(e^{* 2} / \epsilon_{0}\right) \tilde{n}^{2} \pi^{2} \xi^{4} L \ln (L / \xi)$, where $L$ is the length of the vortex line. By equating these values one estimates $\tilde{n}$ as $\sim n / \ln (L / \xi)$, which is macroscopically small. As a result the electric field and vortex profiles are not affected by some redistribution of the normal component due to the electric field.

Finally, let us estimate the size and the electric field of the vortex core by the use of the material parameters typical for oxides such as $m^{*}=10 m_{e}, e^{*}=2 e, n=10^{21} \mathrm{~cm}^{-3}$, and $\epsilon_{0}$ $=10^{3}$. With these parameters I obtain $\xi(0) \simeq 0.48 \mathrm{~nm}$, $\lambda(0) \simeq 265 \mathrm{~nm}$, the Ginsburg-Landau ratio $\kappa \simeq 552$, and $\phi(0) \simeq-8.4 \mathrm{mV}$. Owing to a large dielectric constant the Coulomb repulsion remains weak even for heavy bosons, $r_{s} \simeq 0.46$. The actual size of the charged domain is about $4 \xi$, Fig. 2(a), i.e., about five to ten lattice spacings.

In conclusion, a single vortex problem in the charged Bose liquid has been solved at zero temperature. Differently from the vortex in the BCS superconductors the core of the CBL vortex is charged and there is an electric field inside the core. The charged Bose liquid is an extreme type-II superconductor with the small zero-temperature coherence length, large London penetration depth, and with the sizable electric field of the order of $10 \mathrm{mV}$ or more inside the core. While the magnetic field profile is the same as in the BCS case, the electric field associated with the vortex could provide unique evidence for charged bosons in the high-temperature superconductors. At higher temperatures and (or) in a strong magnetic field we are left with the complicated integrodifferential BdG equations ${ }^{12}$ involving direct and exchange Coulomb interactions of the condensate and "supracondensate" bosons.

The author greatly appreciates useful discussions with C. Dent, A. Junod, N. Hussey, V. Kabanov, and P. Kornilovitch. 
${ }^{1}$ M. R. Schafroth, Phys. Rev. 100, 463 (1955).

${ }^{2}$ L. L. Foldy, Phys. Rev. 124, 649 (1961).

${ }^{3}$ N. Bogoliubov, J. Phys. (Moscow) 11, 23 (1947).

${ }^{4}$ A. L. Fetter, Ann. Phys. (N.Y.) 64, 1 (1971).

${ }^{5}$ R. F. Bishop, J. Low Temp. Phys. 15, 601 (1974).

${ }^{6}$ S. R. Hore and N. E. Frankel, Phys. Rev. B 12, 2619 (1975); 14, 1952 (1976).

${ }^{7}$ D. K. Lee and E. Feenberg, Phys. Rev. 137, A731 (1965).

${ }^{8}$ K. A. Brueckner, Phys. Rev. 156, 204 (1967).

${ }^{9}$ C-W. Woo and S. Ma, Phys. Rev. 159, 176 (1967).

${ }^{10}$ A. S. Alexandrov, Ph.D. thesis, MIFI, Moscow, 1984; Phys. Rev. B 48, 10571 (1993).

${ }^{11}$ A. S. Alexandrov and W. H. Beere, Phys. Rev. B 51, 5887 (1995).

${ }^{12}$ A. S. Alexandrov, W. H. Beere, and V. V. Kabanov, Phys. Rev. B 54, 15363 (1996).

${ }^{13}$ A. P. Mackenzie et al., Phys. Rev. Lett. 71, 1238 (1993); M. S. Osofsky et al., ibid. 71, 2315 (1993); 72, 3292 (1994); A. S. Alexandrov et al., ibid. 76, 983 (1996); D. D. Lawrie et al., J. Low Temp. Phys. 107, 491 (1997); V. F. Gantmacher et al., Zh. Eksp. Teor. Fiz. 115, 268 (1999) [JETP 88, 148 (1999)].

${ }^{14}$ A. Junod, in Studies of High Temperature Superconductors, edited by A. Narlikar (Nova Science, Commack, NY, 1996), Vol. 19, p. 1; A. Junod et al. (unpublished).

${ }^{15}$ A. S. Alexandrov et al., Phys. Rev. Lett. 79, 1551 (1997).

${ }^{16}$ A. S. Alexandrov, Phys. Rev. Lett. 82, 2620 (1999).

${ }^{17}$ A. S. Alexandrov and V. V. Kabanov, Phys. Rev. B 59, 13628
(1999).

${ }^{18}$ A. S. Alexandrov and N. F. Mott, Rep. Prog. Phys. 57, 1197 (1994); High Temperature Superconductors and Other Superfluids (Taylor \& Francis, London, 1994); Polarons and Bipolarons (World Scientific, Singapore, 1995).

${ }^{19}$ A. S. Alexandrov, in Models and Phenomenology for Conventional and High-Temperature Superconductivity, Proceedings of the International School of Physics “Enrico Fermi', Course CXXXVI, Varenna, 1997, edited by G. Iadonisi, J. R. Schrieffer, and M. L. Chiofalo (IOS Press, Amsterdam, 1998), p. 309.

${ }^{20}$ V. L. Ginsburg and L. D. Landau, Zh. Eksp. Teor. Fiz. 20, 1064 (1950).

${ }^{21}$ E. P. Gross, Nuovo Cimento 20, 454 (1961).

${ }^{22}$ L. P. Pitaevskii, Zh. Eksp. Teor. Fiz. 40, 646 (1961) [Sov. Phys. JETP 13, 451 (1961)].

${ }^{23}$ F. London, Superfluids (Wiley, New York, 1950), Vols. I and II.

${ }^{24}$ W. F. Vinen, in Superconductivity, edited by R. D. Parks (Marcel Dekker, New York, 1969), Vol. II, p. 1167.

${ }^{25}$ D. Saint-James, G. Sarma, and E. J. Thomas, Type II Superconductivity (Pergamon, Oxford, 1968).

${ }^{26}$ A. A. Abrikosov, Zh. Eksp. Teor. Fiz. 32, 1442 (1957) [Sov. Phys. JETP 5, 1174 (1957)].

${ }^{27}$ The core of a vortex may also be charged in the Fermi liquid so long as $T_{c} / T_{F}$ is not small ( $T_{F}$ is the Fermi temperature). However, the BCS theory cannot be applied in this case.

${ }^{28}$ L. P. Gor'kov, Zh. Eksp. Teor. Fiz. 36, 1918 (1959) [Sov. Phys. JETP 9, 1364 (1959)]. 